\title{
BMJ Open Impact of mRNA vaccines in curtailing SARS-CoV-2 infection and disability leave utilisation among healthcare workers during the COVID-19 pandemic: cross-sectional analysis from a tertiary healthcare system in the Greater Houston metropolitan area
}

\author{
Farhaan S Vahidy (DD , 1,2,3 Alan P Pan (D) , ${ }^{2}$ Kobina Hagan, ${ }^{2}$ Abdulaziz T Bako, ${ }^{2}$ \\ Henry Dirk Sostman, ${ }^{1,3}$ Roberta L Schwartz, ${ }^{1}$ Robert Phillips, ${ }^{1,2,3}$ Marc L Boom ${ }^{1,3}$
}

To cite: Vahidy FS, Pan AP, Hagan $\mathrm{K}$, et al. Impact of mRNA vaccines in curtailing SARS-CoV-2 infection and disability leave utilisation among healthcare workers during the COVID-19 pandemic: cross-sectional analysis from a tertiary healthcare system in the Greater Houston metropolitan area. BMJ Open 2021;11:e054332. doi:10.1136/ bmjopen-2021-054332

- Prepublication history for this paper is available online. To view these files, please visit the journal online (http://dx.doi. org/10.1136/bmjopen-2021054332).

Received 09 June 2021 Accepted 05 October 2021

Check for updates

(c) Author(s) (or their employer(s)) 2021. Re-use permitted under CC BY-NC. No commercial re-use. See rights and permissions. Published by BMJ.

For numbered affiliations see end of article.

Correspondence to Dr Farhaan S Vahidy; fvahidy@houstonmethodist.org

\section{ABSTRACT}

Objectives We provide an account of real-world effectiveness of COVID-19 vaccines among healthcare workers (HCWs) at a tertiary healthcare system and report trends in SARS-CoV-2 infections and subsequent utilisation of COVID-19-specific short-term disability leave (STDL).

Design Cross-sectional study.

Setting and participants Summary data on 27291 employees at a tertiary healthcare system in the Greater Houston metropolitan area between 15 December 2020 and 5 June 2021. The initial 12-week vaccination programme period (15 December 2020 to 6 March 2021) was defined as a rapid roll-out phase.

Main outcomes and measures At the pandemic onset, HCW testing and surveillance was conducted where SARSCoV-2-positive HCWs were offered STDL. Deidentified summary data of SARS-CoV-2 infections and STDL utilisation among HCWs were analysed. Prevaccination and postvaccination trends in SARS-CoV-2 positivity and STDL utilisation rates were evaluated.

Results Updated for 5 June 2021, 98.2\% ( $n=26791$ ) of employees received a full or partial dose of one of the approved mRNA COVID-19 vaccines. The vaccination rate during the rapid roll-out phase was approximately 3700 doses/7 days. The overall mean weekly SARSCoV-2 positivity rates among HCWs were significantly lower following vaccine roll-out (2.4\%), compared with prevaccination period $(11.8 \%, \mathrm{p}<0.001)$. An accompanying $69.8 \%$ decline in STDL utilisation was also observed (315 to 95 weekly leaves). During the rapid roll-out phase, SARS-CoV-2 positivity rate among Houston Methodist HCWs declined by $84.3 \%$ (8.9\% to $1.4 \%$ positivity rate), compared with a $54.7 \%(12.8 \%$ to $5.8 \%$ positivity rate) decline in the Houston metropolitan area.

Conclusion Despite limited generalisability of regional hospital-based studies - where factors such as the emergence of viral variants and population-level vaccine penetrance may differ-accounts of robust HCW vaccination programmes provide important guidance for
Strengths and limitations of this study

- We tracked COVID-19-specific short-term disability leave utilisation over time among employees of a large tertiary healthcare system in Houston, Texas, which instituted early mandates for COVID-19 vaccination.

- We additionally evaluated data from an employee SARS-CoV-2 surveillance programme with information on COVID-19 vaccination, and symptomatic and breakthrough infections.

- The generalisability of our findings is limited to the setting of our healthcare system.

- Surveillance for SARS-CoV-2 positivity among employees was highly encouraged and testing was readily available across several locations; however, surveillance programme was voluntary.

sustaining a critical resource to provide safe and effective care for patients with and without COVID-19 across healthcare systems.

\section{INTRODUCTION}

Safe and rapid roll-out of the US Food and Drug Administration (FDA)-approved vaccines is a potentially transformational public health tool in the armamentarium against the COVID-19. Despite impressive efficacy data from phase III clinical trials, there is a need to demonstrate real-world effectiveness (RWE) of these vaccines for controlling the pandemic in a variety of settings across heterogenous population groups. Healthcare workers (HCWs) have been on the forefront of the COVID-19 pandemic and continue to provide critical care to hundreds of patients 
with COVID-19 across all US regions and globally. The pandemic has re-established the importance of this valuable resource in being a critically important line of defence against human suffering in the face of a healthcare catastrophe. Most tiered vaccination approaches prioritised HCWs before expanding administration to those in higher risk age groups and with underlying health conditions. This is important from a public health perspective and is critical for continued operational viability of large and small healthcare systems, such that they can adequately provide treatment and prevention services to their communities.

In the state of Texas, vaccine eligibility initially included frontline workers (phase IA) and individuals aged 65 years or older as well as those with underlying health conditions (phase IB). ${ }^{1}$ We provide an account of COVID-19 vaccine roll-out among HCWs of a tertiary healthcare system in Texas. At the time of vaccination programme initiation, the healthcare system was in the midst of a surge in COVID-19 cases and maintaining a viable HCW workforce was critical. ${ }^{12}$ Across the system, a programme of COVID-19-specific short-term disability leave (STDL) among HCWs had been initiated, and tracking its utilisation served as an important indicator of SARS-CoV-2 infection among HCWs and provided a valuable metric to assess the impact of vaccination towards maintaining a healthy HCW workforce. In this report, we demonstrate a signal of RWE of COVID-19 vaccines by evaluating reduction in SARS-CoV-2 infections and subsequent utilisation of STDL among HCWs.

\section{METHODS}

\section{Study design and setting}

Houston Methodist (HM) is an eight-hospital healthcare system in the Greater Houston metropolitan area, which has been a major hub in the fight against the COVID-19 pandemic since March 2020. ${ }^{34}$ At the onset of the pandemic, HM instituted an employee SARS-CoV-2 surveillance initiative coupled with an enhanced COVID19-specific STDL programme for employees testing positive. ${ }^{5}$ Surveillance testing occurred before and after vaccine roll-out and was based on PCR tests for presence of SARS-CoV-2 RNA in nasopharyngeal specimens. The voluntary surveillance programme encouraged all HCWs (symptomatic or asymptomatic) to use SARS-CoV-2 testing at frequent intervals across all HM testing sites. Testing results were typically available in employee health portals within 24-48hours. On the detection of a positive test, employees were required to take STDL and were contacted by supervisors for additional follow-up. Additionally, HM established a system-wide vaccine advisory committee (VAC) in October 2020 to review the safety and efficacy data submitted by vaccine producers to the FDA for consideration of Emergency Use Authorization. The overarching agenda for the VAC was to independently review any available data or guidance before offering vaccines to employees. The VAC subsequently evaluated preliminary data and guidance being provided by the Advisory Committee on Immunization Practices of the Centers for Disease Control and Prevention and developed a risk-based tiered approach for vaccine administration among the employees. Patient-facing HCWs directly involved in the care of patients with COVID-19 were prioritised within the initial weeks after vaccination began on 15 December 2020; all other employees were encouraged to wait until vaccine supply for non-frontline HCWs was available to make an appointment. HM employees received electronic invitations to schedule vaccination across all HM locations and were offered an mRNA vaccine (BNT162b2 or mRNA-1273) being administered on the day of the appointment. During the early stages of vaccine roll-out, various incentives for vaccination were provided and subsequently vaccination was mandated on 7 June 2021. Our analyses represent the premandate time period.

\section{Statistical analysis}

We assimilated deidentified summary data of SARS-CoV-2 infections and STDL utilisation among HCWs across the period of the pandemic and defined the first 12-week period (15 December 2020 to 6 March 2021) as an initial rapid roll-out period for COVID-19 vaccination among HCWs. Summary metrics are provided as frequencies and proportions. Tests for proportional comparisons and $\chi^{2}$ trends were used to assess prevaccination and postvaccination (including the rapid roll-out period) SARS-CoV-2 positivity rates and trends. Reporting of vaccine efficacy was limited to descriptive accounts of the number and proportion of breakthrough infections as assessed through the employee surveillance programme.

\section{Patient and public involvement}

Patients or the public were not involved in the design, or conduct, or reporting, or dissemination plans of our research.

\section{RESULTS}

The COVID-19 vaccine roll-out was simultaneously initiated at all HM locations on 15 December 2020. Updated for 5 June 2021, from among a total of 27291 employees, $26791(98.2 \%)$ had received at least a single dose of either one of the two approved mRNA COVID-19 vaccines, whereas 26723 (97.9\%) had completed both doses. During the 12-week initial rapid roll-out period (15 December 2020 to 6 March 2021) the vaccination rate was 3700 doses $/ 7$ days.

The recent (1 November 2020 to 5 June 2021) trends in SARS-CoV-2 positivity among HCWs are demonstrated in figure 1. The mean SARS-CoV-2 weekly positivity rate prior to initiation of the HCW vaccination programme (11.8\%) was significantly higher compared with the positivity rate following vaccination initiation $(2.4 \%, \mathrm{p}<0.001)$. The upward trend in SARS-CoV-2 positivity rate observed in the 45-day prevaccination period (1 November 2020 to 12 


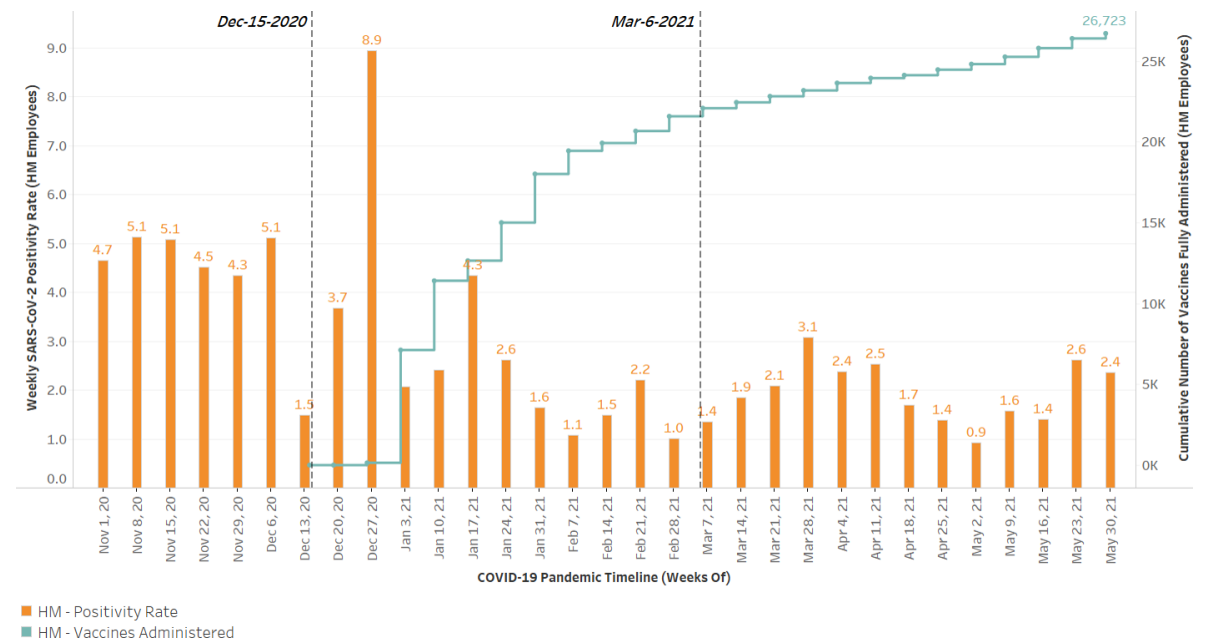

Figure 1 SARS-CoV-2 positivity rates from the Houston Methodist (HM) surveillance employee programme before and after COVID-19 vaccine initiation. Reference lines for the initial 12-week rapid roll-out period for vaccination (15 December 2020 to 6 March 2021) are shown.

December 2020) has significantly trended down during the postvaccination phase (15 December 2020 to 5 June 2021) $\left(\mathrm{p}_{\text {trend }}<0.001\right)$.

Since the end of January 2021, the weekly SARS-CoV-2 infection rate among HCWs participating in surveillance testing has consistently remained below $3.1 \%$. During the initial 12-week rapid roll-out period, the proportional decline in HM HCW SARS-CoV-2 positivity rate was $84.3 \%(8.9 \%$ to $1.4 \%)$, as compared with a $54.7 \%$ decline ( $12.8 \%$ to $5.8 \%)$ observed in the Greater Houston metropolitan area. ${ }^{1}$

As a part of the HCW surveillance programme, 117 $(0.4 \%)$ employees were reported to have tested positive more than 7 days after receiving the second dose of the vaccine, which includes both asymptomatic random surveillance of employees as well as symptomatic referrals from the employee health service. Among these positive cases, $66(56.4 \%)$ were reported to be symptomatic.

Figure 2 represents the weekly frequency of STDL utilisation among HCWs. We also report the approximate temporal emergence of known SARS-CoV-2 mutations $(\mathrm{D} 614 \mathrm{G})^{6}$ as well as detection of viral variants (Alpha B.1.1.7; Beta B.1.351; Gamma P.1 and P.2; B.1.429 and B.1.427) in the Greater Houston area, based on sequencing data of patient specimens performed at $\mathrm{HM}^{7}$ Compared with the peak of STDL utilisation during the

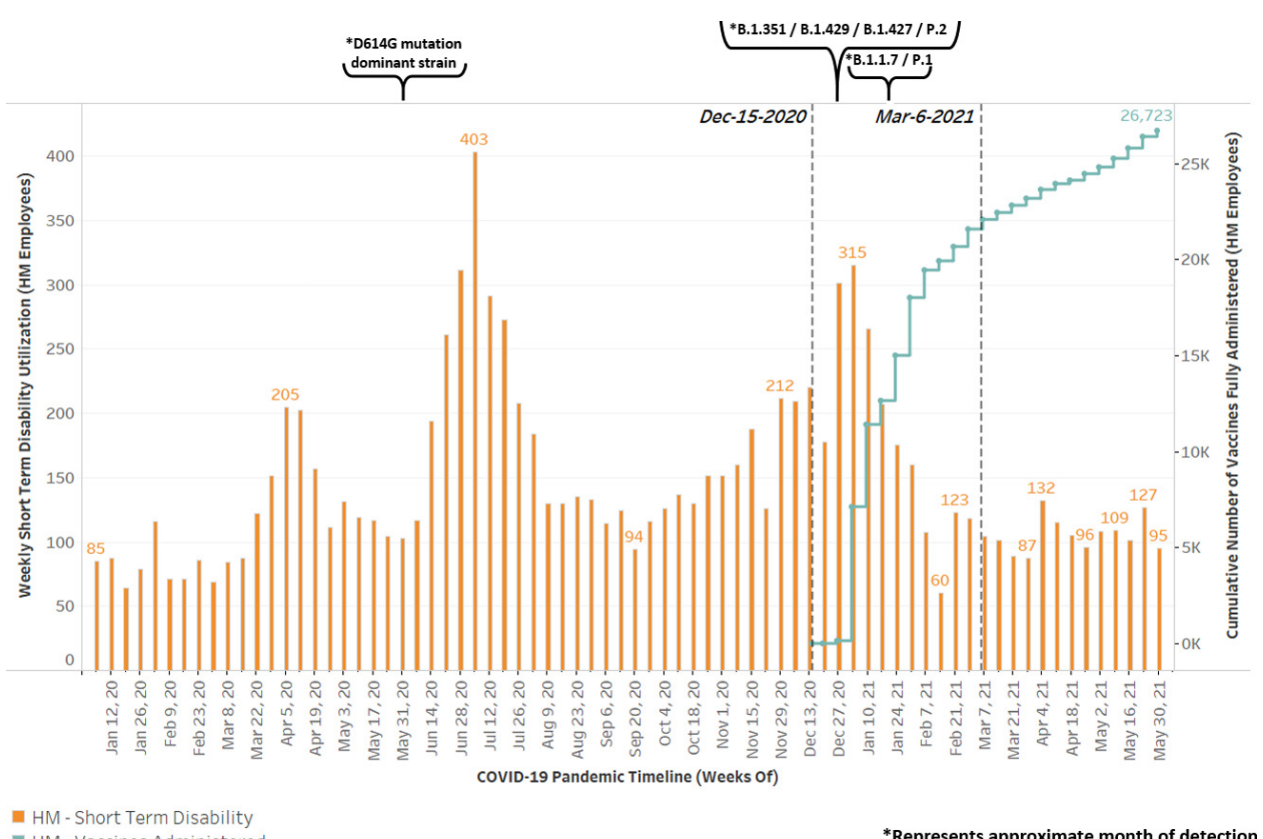

Figure 2 Enhanced short-term disability leave utilisation by Houston Methodist (HM) employees across the COVID-19 pandemic timeline. Reference lines for the initial 12-week rapid roll-out period for vaccination (15 December 2020 to 6 March 2021) are shown. Annotations depict relative emergence and detection of SARS-CoV-2 mutations and viral variants in Greater Houston. 
initial weeks of vaccine roll-out (3-9 January 2021: 315 leaves), a $69.8 \%$ decline has been observed during the most recent reporting period (30 May to 5 June 2021: 95 leaves), with utilisation numbers approaching prepandemic levels.

\section{DISCUSSION}

Given the critical need to maintain an effective and safe healthcare workforce and to minimise inadvertent viral transmission in the healthcare setting, frontline HCWs were ubiquitously recognised as a priority group for vaccination. In addition to continued care provided to patients with and without COVID-19, large healthcare organisations have been called on to organise and execute delivery of COVID-19 vaccines among its HCWs and across the community at large. HM is a state-designated vaccine hub and, as of 5 June 2021, has administered over 780000 vaccines to members of the community. ${ }^{8}$

Vaccine supply and delivery during the initial nationwide roll-out was beset with logistical challenges, and administration metrics lagged behind target levels. However, during the same time period we were able to achieve rapid rates of HCW vaccination with demonstrated vaccine RWE in terms of curtailing infection rates as well as reducing utilisation of STDL for HCWs. Our vaccination planning started several months prior to vaccine delivery and required close coordination between a vaccine scientific committee, operational leadership, physician organisation and the system's infection control management. We instituted a seamless vaccination programme among our HCWs while caring for large numbers of patients with and without COVID-19 and maintaining regular hospital operations. Furthermore, hospital leadership maintained a consistent and transparent line of communication with the workforce. This included weekly communication of the latest scientific and policy updates, reminders on public health guidance and encouragement of individual vaccination. Our initial results with indices of HCW protection against SARSCoV-2 infection and related disability indicate that the vaccines can be deployed in the real-world settings with high levels of effectiveness. Of note, we provide evidence of vaccine effectiveness (VE) amid the emergence of multiple variants of concern (VOC) in the Greater Houston area starting in December 2020.

Interpretation of our findings should take into account the contextual differences between our healthcare system setting and the general Houston public. During the 12-week rapid roll-out period (15 December 2020 to 6 March 2021), vaccines were made available to all HM employees. At the same time, vaccine administration throughout the Greater Houston metropolitan area followed recommendations set by the state of Texas and was only available to frontline workers (phase IA) and individuals aged 65 years and older or with coexisting conditions (phase IB). ${ }^{1}$ Vaccine administration for individuals aged 50 years and older in the general public was not initiated until phase IC (15 March 2021). Given this, it is possible that the phased differences in vaccine eligibility and administration contributed to the observed differences in SARS-CoV-2 positivity rate between the HM workforce and the general Houston population.

Furthermore, it is important to note circumstances influencing the implementation of protective public health measures. Throughout the duration of the pandemic, our hospital system has consistently followed public health recommendations. Personal protective equipment for frontline workers was always made available; masks and social distancing guidelines were followed, even in nonclinical settings. Patients were required to wear masks and the allowance of visitors was restricted, depending on the severity of case surges at the time. Conversely, although a state-wide mask mandate was in effect for a duration of the pandemic (July 2020 to March 2021), adherence to these public health measures may have not been consistently enforced.

Our results are limited to a narrative and descriptive account of the reduction in infection and STDL utilisation across one healthcare system. Furthermore, we have not analysed individual HCW characteristics (such as demographics, comorbidities and risk of occupational exposure) that may be associated with SARS-CoV-2 infection. Concurrent studies on the established immunological protection against infection are needed to fully understand the population-wide and individual benefits that vaccinations confer. The observed trends in SARS-CoV-2 positivity rate were based on diagnostic tests conducted as part of the employee surveillance programme and were requested voluntarily and at the prerogative of the HCW. We did not distinguish results based on either the purpose of testing or whether HCWs experienced viral exposure and/or presented with symptoms. It is possible that the dynamics of programme participation differed in the prevaccination and postvaccination periods. Nonetheless, we observed testing participation to remain relatively consistent during the initial phase of the vaccination programme, with the mean number of weekly tests performed after vaccination roll-out (20 December 2020 to 25 February 2021: 2621 tests) continuing at a rate comparable to that during the peak surveillance period prior to vaccination roll-out (30 August 2020 to 12 December 2020: 2599 tests). Finally, although our data demonstrate a high degree of correlation between vaccination and reduction in infection and STDL utilisation, the potential influence of protective effect offered by lower community spread of the virus or differences in behavioural patterns between health system employees and the general community cannot be ruled out.

Hospitals are a microcosm of the communities they serve as well as a nexus in which there is a high rate of encounters between healthy and ill individuals. Despite determined efforts to vaccinate the broader population, the risk of SARS-CoV-2 infections and related COVID-19 hospitalisations has not been fully eliminated, especially due to the continued emergence of $\operatorname{VOCs}^{9} 10$ and 
relaxation of protective public health measures. Furthermore, although robust VE has been reported, ${ }^{11}$ the duration of VE is currently unknown; consequently, booster shots that confer additional protection against VOCs may be recommended and are currently undergoing testing. ${ }^{12}$

In spite of varying challenges, vaccination rates in the USA have continued at a progressive pace; at the time of this reporting, $\geq 62.9 \%$ of individuals are at least partially immunised and $\geq 53.6 \%$ are fully immunised. ${ }^{13}$ Nonetheless, global vaccination rates are estimated at approximately only $\geq 41.5 \%$ of the population. ${ }^{14}$ As efforts to support international partners in their respective vaccination programmes proceed, insights from the success of vaccine roll-out in the USA will provide a valuable model for reference. Furthermore, in the face of continued high rates of vaccine hesitancy ${ }^{15}$ as well as the risk of a resurgence in cases globally, assimilating and reporting cumulative evidence of real-world VE is paramount to build population-wide confidence in vaccination, hence rapidly achieving desirable levels of herd immunity against the current predominant strains of SARS-CoV-2.

\section{Author affiliations}

${ }^{1}$ Houston Methodist, Houston Methodist Academic Institute, Houston, Texas, USA

${ }^{2}$ Center for Outcomes Research, Houston Methodist, Houston, Texas, USA

${ }^{3}$ Weill Cornell Medicine, New York, New York, USA

Twitter Farhaan S Vahidy @vahidyf and Henry Dirk Sostman @HMAIChief

Acknowledgements We would like to acknowledge the efforts of the following Houston Methodist employees in successfully instituting the employee vaccination and surveillance programmes: Ms Jennifer Borders (System Director Wellness Services), Ms Paula DesRoches (Director Employee Health and Occupational Medicine), Mr Stephen Spielman (SVP and COO of Specialty Physician Group), Mr Jeff Carr (VP Finance) and Dr Dan Metzen (System Director of Pharmacy). All individuals are full-time employees of Houston Methodist and were not financially compensated for this work. We additionally acknowledge all Houston Methodist employees and physicians for their services during the COVID-19 pandemic.

Contributors FSV was responsible for the study conception and design. Data acquisition and analysis were performed by APP. Interpretation of results and initial drafting of the manuscript were completed by FSV and APP. FSV, APP, KH, ATB, HDS, RLS, RP and MLB contributed to critical revision of the manuscript and approved the final version for submission. FSV acted as the study's guarantor.

Funding The authors have not declared a specific grant for this research from any funding agency in the public, commercial or not-for-profit sectors.

Competing interests None declared.

Patient and public involvement Patients and/or the public were not involved in the design, or conduct, or reporting, or dissemination plans of this research.

Patient consent for publication Not required.

Ethics approval This study was not regarded as human subject research by the Houston Methodist (HM) Institutional Review Board (IRB) since this study does not involve direct human participation and was therefore exempt from human subject approval. This study was approved by the HM Institutional Review Board as a quality improvement project with waiver of informed consent.
Provenance and peer review Not commissioned; externally peer reviewed.

Data availability statement Data are available upon reasonable request. Data cannot be shared publicly because of hospital employee confidentiality concerns. Reasonable requests by researchers who meet the criteria for access to confidential data can be made to the corresponding author (fvahidy@houstonmethodist.org).

Open access This is an open access article distributed in accordance with the Creative Commons Attribution Non Commercial (CC BY-NC 4.0) license, which permits others to distribute, remix, adapt, build upon this work non-commercially, and license their derivative works on different terms, provided the original work is properly cited, appropriate credit is given, any changes made indicated, and the use is non-commercial. See: http://creativecommons.org/licenses/by-nc/4.0/.

ORCID iDs

Farhaan S Vahidy http://orcid.org/0000-0002-3464-2111

Alan P Pan http://orcid.org/0000-0002-8782-8024

\section{REFERENCES}

1 Texas Department of State Health Services. Texas COVID-19 data. Available: https://dshs.texas.gov/coronavirus/cases.aspx [Accessed 8 Feb 2021].

2 Texas Medical Center. Coronavirus (COVID-19) updates. Available: https://www.tmc.edu/coronavirus-updates/ [Accessed 8 Feb 2021].

3 Tittle S, Braxton C, Schwartz RL. A guide for surgical and procedura recovery after the first surge of Covid-19. NEJM Catalyst Innovations in Care Delivery. [Epub ahead of print: 2 July 2020]. doi:10.1056/ cat.20.0287

4 Vahidy FS, Drews AL, Masud FN, et al. Characteristics and outcomes of COVID-19 patients during initial peak and resurgence in the Houston metropolitan area. JAMA 2020;324:998.

5 Vahidy FS, Bernard DW, Boom ML, et al. Prevalence of SARS-CoV-2 infection among asymptomatic health care workers in the greater Houston, Texas, area. JAMA Netw Open 2020;3:e2016451.

6 Long SW, Olsen RJ, Christensen PA, et al. Molecular architecture of early dissemination and massive second wave of the SARS-CoV-2 virus in a major metropolitan area. MBio 2020;11.

7 Long SW, Olsen RJ, Christensen PA, et al. Sequence analysis of 20,453 severe acute respiratory syndrome coronavirus 2 genomes from the Houston metropolitan area identifies the emergence and widespread distribution of multiple isolates of all major variants of concern. Am J Pathol 2021;191:983-92.

8 Houston Methodist. COVID-19 vaccine information. Available: https://www.houstonmethodist.org/coronavirus/vaccine-updates/ [Accessed 7 Mar 2021].

9 Walensky RP, Walke HT, Fauci AS. SARS-CoV-2 variants of concern in the United States-Challenges and opportunities. JAMA 2021;325:1037.

10 Mascola JR, Graham BS, Fauci AS. SARS-CoV-2 viral VariantsTackling a moving target. JAMA 2021;325:1261.

11 Pilishvili T, Fleming-Dutra KE, Farrar JL, et al. Interim Estimates of Vaccine Effectiveness of Pfizer-BioNTech and Moderna COVID-19 Vaccines Among Health Care Personnel - 33 U.S. Sites, JanuaryMarch 2021. MMWR Morb Mortal Wkly Rep 2021;70:753-8.

12 Rubin R. COVID-19 vaccines vs Variants-Determining how much immunity is enough. JAMA 2021;325:1241.

13 Centers for Disease Control and Prevention. COVID-19 vaccinations in the United States. COVID data Tracker. Available: https://covid. cdc.gov/covid-data-tracker/\#vaccinations [Accessed 1 Jun 2021].

14 Mathieu E, Ritchie H, Ortiz-Ospina E. A global database of COVID-19 vaccinations. Nat Hum Behav. [Epub ahead of print: 10 May 2021]. doi:10.1038/s41562-021-01122-8

15 University of Houston Hobby School of Public Affairs. Texas Policy \& Politics 2021: Texans and the COVID-19 Vaccine. Available: https:// uh.edu/hobby/tx2021/ [Accessed 3 Feb 2021]. 